\title{
Visualizing the origin of rotational entropy effects in coadsorbed systems
}

\author{
Ryan T. Hannagan, ${ }^{1}$ Kyle Groden, ${ }^{2}$ Amanda M. Larson, ${ }^{1}$ Andrew J. Therrien, ${ }^{1}$ Theodore Thuening, ${ }^{1}$ \\ Alex C. Schilling, ${ }^{1}$ Jean-Sabin McEwen, ${ }^{2,3,4,5,6,{ }^{*}}$ and E. Charles H. Sykes ${ }^{1, *}$ \\ ${ }^{1}$ Department of Chemistry, Tufts University, Medford, Massachusetts 02155, USA \\ ${ }^{2}$ The Gene and Linda Voiland School of Chemical Engineering and Bioengineering, \\ Washington State University, Pullman, Washington 99164, USA \\ ${ }^{3}$ Department of Chemistry, Washington State University, Pullman, Washington 99164, USA \\ ${ }^{4}$ Department of Physics, Washington State University, Pullman, Washington 99164, USA \\ ${ }^{5}$ Department of Biological Systems Engineering, Washington State University, Pullman, Washington 99164, USA \\ ${ }^{6}$ Institute of Integrated Catalysis, Pacific Northwest National Laboratory, Richland, Washington 99354, USA
}

(Received 3 May 2019; accepted 18 May 2020; published 12 June 2020)

\begin{abstract}
We demonstrate that significant entropic effects govern the stability of rotationally constrained molecular complexes on surfaces. Using a complementary combination of low-temperature scanning tunneling microscopy, temperature programmed desorption, and density functional theory calculations, we probe the energetics of surface adsorbed molecular rotors as well as their corresponding rotationally hindered complexes. The results reveal that absorbate-absorbate interactions can significantly decrease the rotational entropy associated with such complexes leading to lower than expected desorption temperatures.
\end{abstract}

DOI: 10.1103/PhysRevResearch.2.023326

\section{INTRODUCTION}

The interactions between molecules on surfaces is of fundamental interest due to their relevance to many research areas, such as heterogeneous catalysis, semiconductors, corrosion inhibition, and lubrication [1]. For many years, these interactions could only be probed experimentally due to the complexity of coupling molecule-molecule with molecule surface interactions. Modern computational architecture has now made it possible to efficiently quantify the energetics of molecular adsorption and their interactions from fundamental quantum mechanical principles [2-4]. Despite these advances, subsequent analysis of these results often neglects entropic effects that can play an important role in the fundamental physics of the systems under examination. Kreuzer and Payne have shown how the desorption rate depends on the chemical potential of the adsorbate under quasiequilibrium conditions and thus entropy must be considered. Under simplifying assumptions (see Appendix), these results can be reduced to the familiar Polanyi-Wigner equation $[5,6]$. Recent work by Campbell and co-workers has shown that, for simple molecular desorption, these entropic effects can often be more significant than expected [7-9]. For instance, Campbell and Sellers found the entropy of many molecules bound to surfaces can be nearly two-thirds of the gas phase entropy [9]. Significant entropic effects have also been observed by

\footnotetext{
*Corresponding authors: js.mcewen@wsu.edu; Charles.Sykes@tufts.edu

Published by the American Physical Society under the terms of the Creative Commons Attribution 4.0 International license. Further distribution of this work must maintain attribution to the author(s) and the published article's title, journal citation, and DOI.
}

Ellis and co-workers who observed entropically enhanced diffusion of benzene on $\mathrm{Cu}(001)$ [10]. The importance of these entropic contributions to diffusion are relevant even at cryogenic temperatures [11]. Campbell and co-workers have discussed models that account for entropic contributions from vibration, rotation, and translation, but they restrict their discussion to systems with isolated molecular desorption without considering absorbate-absorbate interactions $[12,13]$.

While the important role of rotational entropy has been addressed for molecular desorption, the influence of absorbateabsorbate interactions on adsorbate rotational motion is still unknown. Since many processes of fundamental importance are governed by the interactions between coadsorbed molecules, it is pertinent to consider the more complicated case where absorbate-absorbate interactions are present. Bradshaw and co-workers studied the behavior of $\mathrm{CO}$ complexes on $\mathrm{Cu}(110)$ and interestingly found that $\mathrm{CO}$ dimers diffused faster than $\mathrm{CO}$ monomers due to different diffusion preexponential factors [14]. Although entropic contributions have been examined for small molecules such as CO $[15,16]$, examination of these contributions for more complex molecules has been largely absent in the literature. In this study, we present a combined low-temperature scanning tunneling microscope (LT-STM), temperature programed desorption (TPD), and density functional theory (DFT) study of propylene oxide (PO) and propylene (Py) complexes on $\mathrm{Cu}(111)$. These complementary techniques allow us to both visualize the dynamic motion of molecules and quantify the energetics, thereby developing a physically meaningful DFT-based model to understand how an attractive molecular interaction between adsorbates can be counteracted by the effect of rotational entropy at elevated temperatures. Our LT-STM results show these molecules form 1:1 complexes comprised of $1 \mathrm{Py}$ and $1 \mathrm{PO}$ molecules due to enthalpic attractions, a result confirmed by our DFT calculations which 


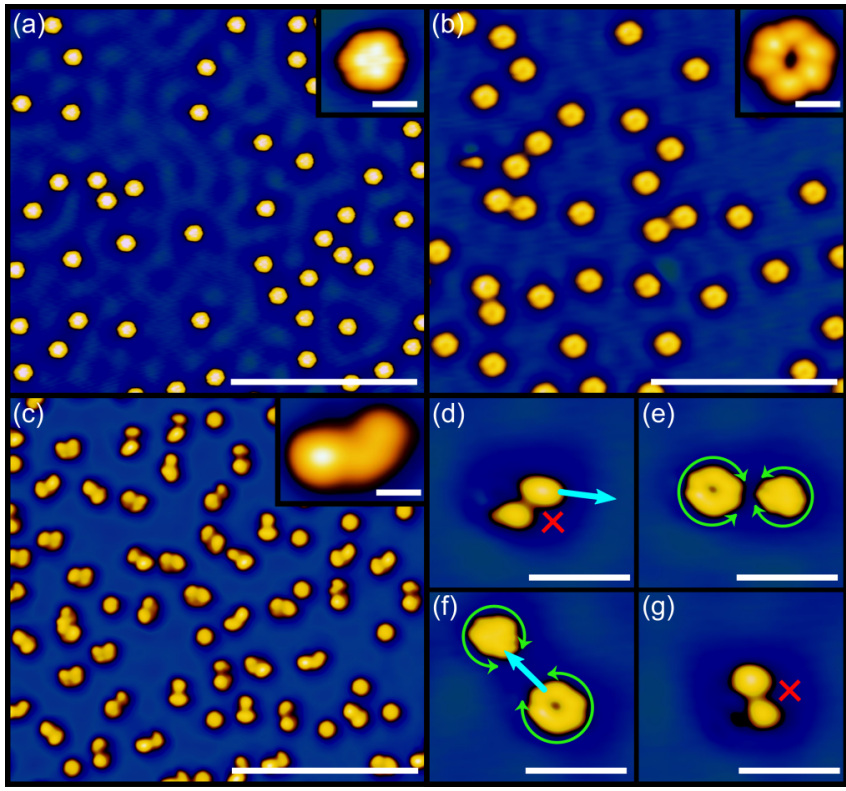

FIG. 1. LT-STM images showing how the attractive lateral interactions between rotating PO and Py molecules lead to rotationally constrained complexes when they are coadsorbed on a $\mathrm{Cu}(111)$ surface. (a) STM image depicting the rotation of pure Py, (b) pure $\mathrm{PO}$, and (c) static 1:1 complexes. Insets shows high resolution images of each molecule. Panels (d)-(g) show molecular manipulation experiments in which we are able to start and stop rotations using STM tip manipulation. Manipulation paths are shown with blue arrows. Panel (d) shows a PyPO complex which is pulled apart resulting in two rotationally free molecules as seen in (e). Panel (f) shows two rotational free molecules which are pushed together to form a rotationally constrained complex as seen in $(\mathrm{g})$. The green arrows highlight rotating molecules while the red crosses highlight stationary complexes. All images were collected at $5 \mathrm{~K}$. Typical imaging conditions were $+/-60 \mathrm{mV}$ and tunneling currents $<70 \mathrm{pA}$. Scale bars are $10 \mathrm{~nm}$ in main figure panels (a)-(c), $5 \mathrm{~nm}$ in figure panels (d) $-(\mathrm{g})$, and $0.5 \mathrm{~nm}$ in the insets.

show an attractive interaction between $\mathrm{Py}$ and $\mathrm{PO}$ of $-0.14 \mathrm{eV}$ [17]. However, our TPD results show that there is no net stabilization of Py by PO, which means entropy must play an important role. Using a DFT and statistical mechanical model, we show that, around the desorption temperature, some of the PyPO complexes break apart due to the increase in entropy associated with breaking a rotationally constrained complex into two rotationally free monomers. Therefore, these results demonstrate how absorbate-absorbate interactions can influence the entropy of the system and demonstrate that attractive interactions between rotationally free molecules can lead to a decrease in entropy due to changes in rotational degrees of freedom. To accurately analyze desorption rates from surfaces, these effects must be accounted for.

\section{EXPERIMENTAL RESULTS AND THEORETICAL MODELING}

Figure 1 shows a series of STM images of Py and PO on $\mathrm{Cu}(111)$. Both Py [Fig. 1(a)] and PO [Fig. 1(b)] display net repulsive interactions between like molecules that prevent the formation of large agglomerates even after annealing $>40 \mathrm{~K}$ to equilibrate the system. The isolated Py and PO molecules freely rotate on the surface, which results in the appearance of a sixfold symmetric shape due to time-averaged STM imaging of the rotating molecule, as seen in the insets of Figs. 1(a) and 1(b). In contrast to the isolated behavior, when Py and PO are coadsorbed on the surface and equilibrated, they form 1:1 complexes as seen in Fig. 1(c). Formation of the complexes, however, leads to a loss of rotational freedom for both molecules as seen in the inset [17]. Using the STM tip, we can pull the complexes apart to identify them primarily as dimer complexes comprised of one Py and one PO molecule. As seen from the sixfold rotational symmetry in panels (a) and (b) both Py and PO are free to rotate when they are isolated from one another but are stationary in the complex. We show examples of STM tip induced single molecule manipulations in which we are able to start and stop molecular rotation in Fig. 1, panels (d)-(g). In panel (g) we use the tip to pull apart a stationary complex and form two rotationally free molecules as seen in panel (e). In panel (f) we use the STM tip to push two molecules back together and rotation is stopped due to complex formation as seen in panel $(\mathrm{g})$. Complex formation, being spontaneous when the Py/PO system is equilibrated, must therefore be enthalpically favored as complexation results in loss of rotational entropy. This is supported by our DFT-based models that indicate the enthalpic gain due to complexation is $-0.14 \mathrm{eV}$ [17].

Figure 2 shows a series of TPD traces collected with increasing coverages of PO [Fig. 2(a)] and Py [Fig. 2(b)] on $\mathrm{Cu}(111)$. The insets show the change in desorption energy as a function of initial coverage computed via a Redhead analysis [18]. Preexponentials were calculated using available gas phase entropy data and the correlations presented by Campbell and Sellers, resulting in a preexponential on the order of $\sim 10^{15} \mathrm{~s}^{-1}$ for both molecules [8,9,19,20]. For PO, we observe a monolayer peak $(\alpha)$ and a multilayer peak $(\beta)$ in good agreement with previous work by Gellman and co-workers who have studied PO on $\mathrm{Cu}$ surfaces [21,22]. For Py desorption, we again observe a monolayer peak $(\alpha)$ and a multilayer peak $(\beta)$. Similar desorption features are reported by Gellman and White who collected TPD and vibrational spectra of $\mathrm{Py}$ on $\mathrm{Cu}(111)$ and $\mathrm{Ag}(111)$, respectively [23-25]. As expected from our STM data, both Py and PO display repulsive interactions, which we quantified by the TPD analysis shown in the insets in Fig. 2. A nearly linear decrease in desorption energy from the low coverage limit to around 0.5 ML is observed. In the zero-coverage limit, the experimental desorption energies of isolated PO and Py molecules are $0.58 \mathrm{eV}$ and $0.44 \mathrm{eV}$, respectively, and are in very good agreement with our corresponding DFT-based values of $0.5 \mathrm{eV}$ and $0.4 \mathrm{eV}$ [17].

To quantify the desorption energetics of the dimer complex phase we observed via STM, we conducted a series of coadsorption TPD experiments in which a mixture of Py and PO were exposed to the surface. TPD is quite sensitive to the lateral interactions between adsorbates, even on the order of a few meV [21]. Figure 3(a) shows a representative coadsorption experiment along with solo-dose controls for comparison. Initial coverages of $\mathrm{Py}$ and $\mathrm{PO}$ in this experiment were both 0.014 molecules per $\mathrm{Cu}$ atom. Guided by the STM and DFT results that show evidence of attractive lateral interactions 

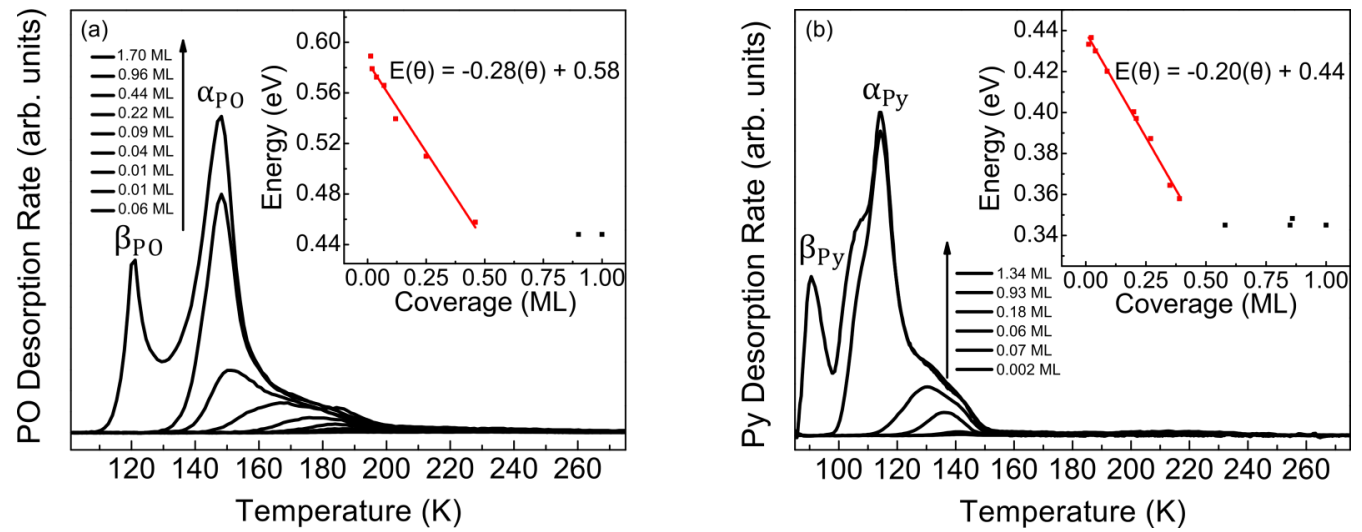

FIG. 2. TPD traces showing desorption of various initial coverages of (a) propylene oxide (PO) and (b) propylene (Py) from Cu(111). The insets show the change in the desorption energy as a function of initial coverage for both PO and Py, respectively, revealing that both PO and Py display repulsive lateral interactions below $\sim 0.5 \mathrm{ML}$. Coverages are reported here with respect to the saturated monolayer (ML) of each molecule.

between PO and Py [17], one may expect Py to desorb at a higher temperature when complexed with PO. Counterintuitively, Py desorbs at a slightly lower temperature after coadsorbed with PO as compared to the corresponding experiment where the same initial coverage of Py was present on the surface. If we account for the increase in local coverage of $\mathrm{Py}$ in the presence of PO by comparing to an equivalent total coverage (initial coverage of Py plus PO), we see nearly identical peak desorption temperatures, i.e., no net stabilization due to complexation. This lack of stabilization is shown for a series of coadsorption TPD experiments as seen in Fig. 3(b). The data for the desorption of Py in the presence of PO falls within the same range as desorption of isolated Py and we do not find a significant difference between the two data sets. This is despite an enthalpic stabilization of the complex of $-0.14 \mathrm{eV}$ according to DFT [17]. We estimate a stabilization of this magnitude for the PyPO complex (assuming no change in preexponential) would result in a shift of $40 \mathrm{~K}$ in the corresponding TPD spectra.

This result is particularly surprising if one draws an analogy to the desorption of Py from the PyPO complex with the desorption of CO from stepped surfaces. Since CO molecules on a stepped metal surface can be safely assumed to be in quasiequilibrium during a TPD experiment (i.e., the time scale for $\mathrm{CO}$ desorption is much larger than the time scale for diffusion), the desorption of a more strongly bound $\mathrm{CO}$ at a step site will occur at a higher temperature as compared to desorption of $\mathrm{CO}$ from a terrace site $[26,27]$. The fact that we do not observe this when Py desorbs from a mixture of PO and $\mathrm{Py}$ on $\mathrm{Cu}(111)$ is strong evidence that entropic effects, which are typically more relevant at higher temperatures, play a significant role. To further elucidate these results, we constructed a free energy based model using DFT-based calculations and statistical mechanics.
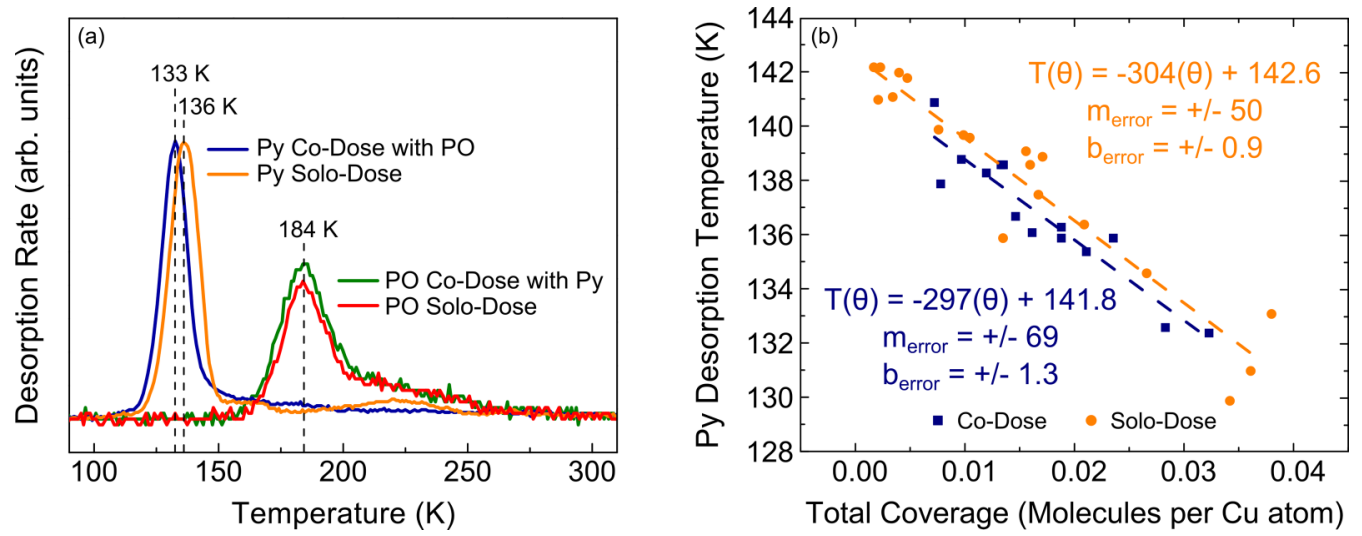

FIG. 3. Coadsorption experiments in which the surface was exposed to a mixture of Py and PO showing no significant difference in peak desorption temperature for isolated Py, and Py in the presence of PO when total coverage is accounted for. (a) Representative solo and coadsorption TPD experiments. The low temperature peak at $133 \mathrm{~K}$ is Py desorption in the presence of PO, while the peak at $136 \mathrm{~K}$ is isolated Py desorption. High temperature peaks at $184 \mathrm{~K}$ are PO desorption in both cases showing no temperature difference. The slightly lower desorption temperature of Py when codosed with PO is due to the increase in local coverage caused by the adsorbed PO. (b) Graph showing the change in Py desorption temperature as a function of total initial coverage for the series of coadsorption experiments (blue) as compared to control experiments with only Py (orange). After accounting for total coverage, there is no significant difference between the Py only and codose data. Reported errors are at $95 \%$ confidence intervals. 
As we previously mentioned and outlined in the Appendix, we consider the chemical potentials of both the isolated molecules and the complex (assuming no lateral interactions, or that the configurational integral is zero) to provide insights into the complex and monomer distribution during the TPD experiments. As such, we take the following reaction representing the complexation of Py and PO on the surface (where all species are surface-bound):

$$
\mathrm{Py}^{*}+\mathrm{PO}^{*} \leftrightarrow \mathrm{PyPO}^{*} .
$$

The quasiequilibrium criterion for this surface reaction can be described in terms of the chemical potentials of these species,

$$
\mu_{\mathrm{PyPO}}\left(T, \theta_{\mathrm{PyPO}}\right)-\mu_{\mathrm{Py}}\left(T, \theta_{\mathrm{Py}}\right)-\mu_{\mathrm{PO}}\left(T, \theta_{\mathrm{PO}}\right)=0,
$$

where the chemical potential of the s species, $\mu_{\mathrm{s}}\left(T, \theta_{\mathrm{s}}\right)$, is a function of the temperature $T$ and the coverage of the species $\theta_{\mathrm{s}}$ (on a per surface atom basis). Note that each PyPO complex is counted as one molecule in this definition (see the Appendix for more details). We also remark that by assuming the quasiequilibrium criterion given in Eq. (1), we are also assuming that the time scale for dissociating the PyPO complex in this process, $\tau_{\text {complex }}$, must be much shorter than the time scale for desorption, $\tau_{\text {des }}$. It also assumes that the time scales for diffusion of the PO and the Py monomers, $\tau_{\text {diff }}$, are much shorter than the time scale for desorption. If either of these conditions were not satisfied ( $\tau_{\text {complex }} \ll \tau_{\text {des }}$ or $\tau_{\text {diff }} \ll \tau_{\text {des }}$ ), then we could not model using the formalism described in the Appendix and would need to model the desorption kinetics with a kinetic lattice gas model [28-30].

We have derived these chemical potentials in the Appendix and, using these definitions, the equilibrium criterion can be expanded to

$$
\begin{aligned}
& \Delta E_{\text {complex }}-k_{\mathrm{B}} T \ln \left(\frac{\sqrt{\Theta_{\mathrm{R}, \mathrm{PO}} \Theta_{\mathrm{R}, \mathrm{Py}}}}{\pi T} \frac{\frac{2 \pi m_{\mathrm{P} \mathrm{PO}} k_{\mathrm{B}} T}{h^{2}}\left(\frac{\sqrt{3}}{2} a^{2}\right)}{\chi_{\mathrm{PO}}(T) \cdot \chi_{\mathrm{Py}}(T)}\right) \\
& +k_{\mathrm{B}} T \ln \left(\frac{\theta_{\mathrm{PyPO}}}{\theta_{\mathrm{Py}} \theta_{\mathrm{PO}}}\right)=0 .
\end{aligned}
$$

In this equation, $\Delta E_{\text {complex }}$ is the electronic energy of complexation defined as $\Delta E_{\text {complex }}=\Delta E_{\text {ads,PyPO }}-\Delta E_{\text {ads, } \mathrm{PO}}-$ $\Delta E_{\text {ads,Py }}$, where $\Delta E_{\text {ads,PyPO }}$ is the adsorption energy of the $\mathrm{PyPO}$ complex on $\mathrm{Cu}(111), \Delta E_{\text {ads, } \mathrm{PO}}$ is the adsorption energy of $\mathrm{PO}$ on $\mathrm{Cu}(111)$, and $\Delta E_{\text {ads,Py }}$ is the adsorption energy of $\mathrm{Py}$ on $\mathrm{Cu}(111)$ (all with respect to isolated PO and/or Py in the gas phase). Further, $\Theta_{R, P O}$ and $\Theta_{R, P y}$ are the rotational temperatures of the molecules on the surface, $\chi_{\mathrm{PO}}(T)$ and $\chi_{\mathrm{Py}}(T)$ are functions for the hindered translator treatment of the adsorbates when not complexed (see the Appendix for the definition), $m_{\mathrm{PyPO}}$ is the mass of the PyPO complex, and $a$ is the $\mathrm{Cu}-\mathrm{Cu}$ spacing on the $\mathrm{Cu}(111)$ surface. We can define a "standard" change in Gibbs energy of complexation (i.e., if all coverages were unity), $\Delta G_{\text {complex }}^{\circ}(T)$, as

$$
\begin{aligned}
& \Delta G_{\text {complex }}^{\circ}(T) \\
& \quad=\Delta E_{\text {complex }}-k_{\mathrm{B}} T \ln \left(\frac{\sqrt{\Theta_{\mathrm{R}, \mathrm{PO}} \Theta_{\mathrm{R}, \mathrm{Py}}}}{\pi T} \frac{\frac{2 \pi m_{\mathrm{PyPO}} k_{\mathrm{B}} T}{h^{2}}\left(\frac{\sqrt{3}}{2} a^{2}\right)}{\chi_{\mathrm{PO}}(T) \cdot \chi_{\mathrm{Py}}(T)}\right),
\end{aligned}
$$

making the equilibrium criterion simply

$$
\Delta G_{\text {complex }}^{\circ}(T)+k_{\mathrm{B}} T \ln \left(\frac{\theta_{\mathrm{PyPO}}}{\theta_{\mathrm{Py}} \theta_{\mathrm{PO}}}\right)=0 .
$$

It is clear that this standard Gibbs energy of complexation is made up of electronic $\left(\Delta G_{\text {elec }}^{\circ}\right)$, translational $\left[\Delta G_{\text {trans }}^{\circ}(T)\right]$, and rotational $\left[\Delta G_{\text {rot }}^{\circ}(T)\right]$ components

$$
\Delta G_{\text {complex }}^{\circ}(T)=\Delta G_{\text {elec }}^{\circ}+\Delta G_{\text {trans }}^{\circ}(T)+\Delta G_{\text {rot }}^{\circ}(T),
$$

where these components are defined as

$$
\begin{gathered}
\Delta G_{\text {elec }}^{\circ}=\Delta E_{\text {complex }}, \\
\Delta G_{\text {trans }}^{\circ}(T)=-k_{\mathrm{B}} T \ln \left(\frac{\frac{2 \pi m_{\mathrm{PypO}} k_{\mathrm{B}} T}{h^{2}}\left(\frac{\sqrt{3}}{2} a^{2}\right)}{\chi_{\mathrm{PO}}(T) \cdot \chi_{\mathrm{Py}}(T)}\right), \\
\Delta G_{\mathrm{rot}}^{\circ}(T)=-k_{\mathrm{B}} T \ln \left(\frac{\sqrt{\Theta_{\mathrm{R}, \mathrm{PO}} \Theta_{\mathrm{R}, \mathrm{Py}}}}{\pi T}\right) .
\end{gathered}
$$

This splitting of the standard Gibbs energy change into components allows us to understand how each contribution affects the equilibrium distribution of each species on the surface.

Consulting Eq. (5), we can solve for the equilibrium coverages of the Py and PO monomers and PyPO clusters at a given temperature. If we assume the coverage of each monomer is initially $\theta_{\exp }$ and to attain equilibrium it must decrease by an arbitrary amount $x$, Eq. (5) becomes

$$
\frac{x}{\left(\theta_{\exp }-x\right)^{2}}=\exp \left(-\frac{\Delta G_{\text {complex }}^{\circ}(T)}{k_{\mathrm{B}} T}\right) .
$$

Solving this for $x$ gives us the coverages of monomers and clusters at equilibrium as a function of temperature, as shown in Fig. 4.

These results demonstrate that, even though there is an enthalpic interaction favoring complexation, an equilibrated surface at the desorption temperature will consist of a distribution of monomers and complexes due to the low entropy of the complexed state. The rotational contribution to the standard Gibbs energy plays an important role here, as it provides a constant, positive penalty to complexation that negates the enthalpic stability of the complexes. This is further demonstrated by the coverage distributions in Fig. 4 when this rotational contribution to the standard Gibbs complexation energy is zero, as only complexes are expected on the surface. Physically, the monomeric phase contains a higher number of states (due to the degrees of freedom of the free rotating monomer) that can be occupied as one increases the temperature as compared to the complexed phase that is rotationally arrested. Thus, as temperature is increased, the occupation of these rotational states becomes favored in comparison to the enthalpic interaction experienced through complexation.

Our equilibrium calculations (Fig. 4) show that we would expect a mixture of monomer Py and PyPO complexes at the experimental desorption temperature. This raises the following question: if there are two states present, why do we not observe two discreate desorption features? While our DFT model predicts the attractive interaction between $\mathrm{Py}$ and $\mathrm{PO}$ to be $-0.14 \mathrm{eV}$ [17], which should correspond to a $\sim 40 \mathrm{~K}$ higher desorption temperature of Py from the complex relative to the isolated molecules, this calculation neglects any entropic 

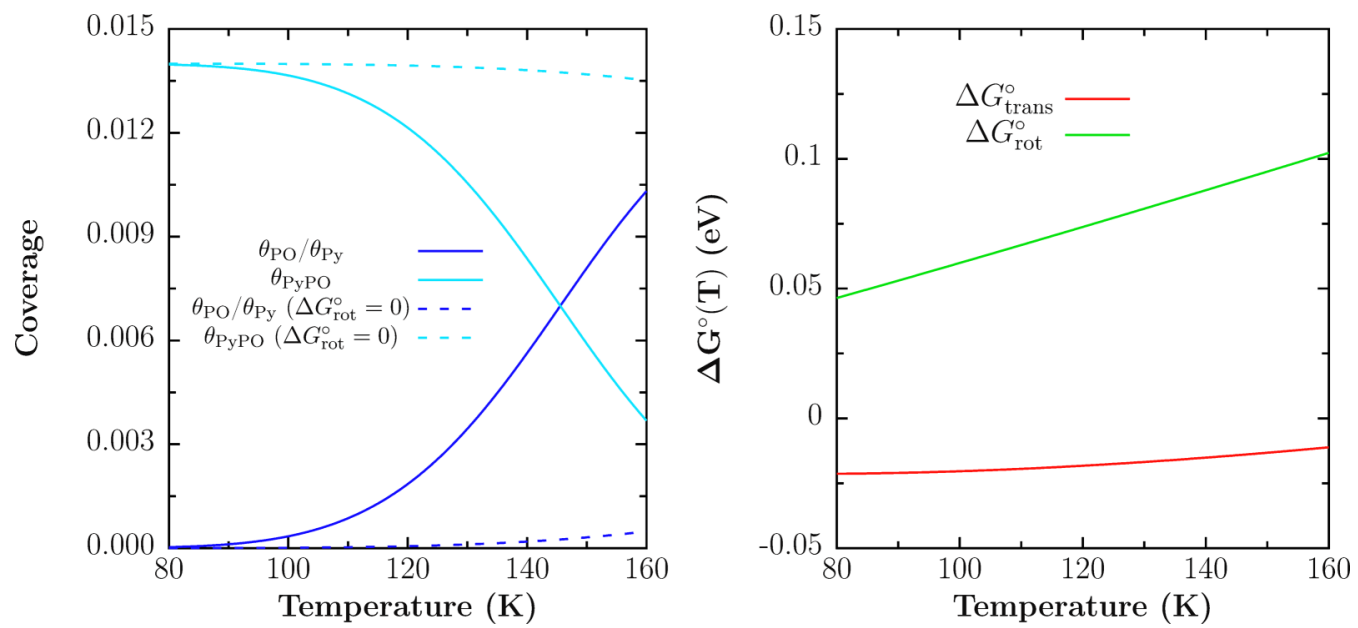

FIG. 4. Coverages of the PO and Py monomers and the PyPO complex as a function of temperature (left). Note that the case where there is no rotational contribution to the standard Gibbs energy of complexation is shown for comparison as dashed lines. Additionally, the translational and rotational contributions to the standard Gibbs energy of the complex are plotted as a function of temperature for comparison (right).

effects which clearly play an important role in this system. First order molecular desorption from a metal surface is often considered using the Polanyi-Wigner equation, which gives the rate of desorption as a function of the preexponential, desorption energy, coverage, and temperature. As shown by transition state theory [31], the preexponential term depends on entropy; therefore, we expect entropy effects to manifest themselves in the desorption kinetics of this system. As we show in the Appendix, this can also be expressed in terms of chemical potential. The reason we only observe one feature in the desorption experiment despite there being two states present must be the free energy of desorption is the same for monomer Py and Py in the PyPO complex. Put another way, the entropic payback of breaking the complex negates the $0.14 \mathrm{eV}$ enthalpic stabilization predicted by DFT at the desorption temperature so the free energy of desorption from the complex is equal to the free energy of desorption of the monomer.

\section{CONCLUSIONS}

In conclusion, we have determined how rotational entropy effects dictate the stability of molecular complexes on surfaces and how this impacts the resulting desorption kinetics. LT-STM data indicates that Py and PO display attractive interactions and DFT based calculations, which alone neglect entropic effects, predict this attractive interaction to be approximately $-0.14 \mathrm{eV}$ [17]. However, TPD experiments result in identical desorption temperatures for isolated and complexed Py molecules. Our statistical mechanical modeling reveals that both PyPO complexes and isolated Py exist at the desorption temperature and the dissociation of the complex is driven by an increase of rotational entropy. The fact that Py desorbs from the surface whether or not PO is present indicates that the entropy of complex formation (bringing two rotationally free molecules together to form a rotationally locked complex) negates the enthalpic interaction at the desorption temperature, resulting in almost identical free energies of desorption for isolated Py and Py from the PyPO complex. The current work goes beyond previous studies of entropic contributions to molecular desorption energies in that adsorbate-adsorbate interactions are considered. We demonstrate that the reduction of rotational entropy due to the formation of molecule-molecule complexes can have a significant impact on the desorption kinetics. These results should be generalizable to a wide range of interacting molecular systems on surfaces and enable better quantification of important desorption events in, for example, catalytic reactions.

\section{ACKNOWLEDGMENTS}

The authors thank B. Kay, G. Kimmel, and R. Réocreux for useful conversations. Financial support for the experimental work at Tufts was provided by the National Science Foundation under Grant No. CHE-1764270. R.T.H. was supported by the Division of Chemical Sciences, Office of Basic Energy Sciences, CPIMS Program, U.S. Department of Energy, under Grant No. DE-SC0004738. Financial support for the theory work at WSU was provided by the National Science Foundation CAREER program under Contract No. CBET-1653561. A portion of the computer time for the computational work was performed using EMSL, a national scientific user facility sponsored by the Department of Energy's Office of Biological and Environmental Research and located at PNNL. PNNL is a multiprogram national laboratory operated for the US DOE by Battelle.

\section{APPENDIX: VISUALIZING THE ORIGIN OF ROTATIONAL ENTROPY EFFECTS IN COADSORBED SYSTEMS}

\section{Desorption under quasiequilibrium conditions}

In this section, we demonstrate how the desorption rate depends on the chemical potential of the adsorbate, $\mu_{a}$. We first consider the desorption from an adsorbate where the diffusion is so fast (on the time scale of desorption) that the adsorbate is maintained in quasiequilibrium throughout the desorption process. Thus the adsorbate can be characterized by its chemical potential $\mu_{a}(\theta(t), T(t))$. As a next step, we look at a situation where the gas phase pressure of a molecular species, $P$, is different from its value, $\bar{P}$, which 
maintains a coverage $\theta$. As such, to reestablish equilibrium between the gas phase and the adsorbate there is an excess flux that is given by

$$
\frac{d \theta}{d t}=S(\theta, T)(P-\bar{P}) \frac{a_{s} \lambda_{\mathrm{th}}}{h},
$$

where $S(\theta, T)$ is the coverage and temperature-dependent sticking coefficient, $a_{s}$ is the adsorption site area, $h$ is Planck's constant, and

$$
\lambda_{\mathrm{th}}=\frac{h}{\sqrt{2 \pi m k_{\mathrm{B}} T}}
$$

is the thermal wavelength, where $m$ is the mass of the adsorbing species and $k_{\mathrm{B}}$ is Boltzmann's constant. As a next step, we express the equilibrium pressure in terms of the gas phase chemical potential, $\mu_{g}$,

$$
\bar{P}=\frac{k_{\mathrm{B}} T}{\lambda_{\text {th }}^{3}} Z_{\text {int }} \exp \left(\frac{\mu_{g}}{k_{\mathrm{B}} T}\right) .
$$

Here, $Z_{\text {int }}$ is the intramolecular partition function that accounts for rotations and vibrations of the molecule in the gas phase. Further, for nondissociative adsorption, the chemical potential of the adsorbate, $\mu_{a}$, is equal to the chemical potential of the adsorbate in the gas phase at equilibrium. Therefore, the desorption rate can written as

$$
R_{\mathrm{d}}=S(\theta, T) \frac{a_{s}}{\lambda_{\text {th }}^{2}} \frac{k_{\mathrm{B}} T}{h} Z_{\text {int }} \exp \left(\frac{\mu_{a}}{k_{\mathrm{B}} T}\right) .
$$

This is our principal result that shows that the desorption rate directly correlates to the chemical potential of the adsorbate.

One can further show that (S4) reduces down to the familiar Polanyi-Wigner equation for first order desorption if further simplifying assumptions are made. As a first additional assumption, we consider a noninteracting adsorbate in the submonolayer regime, for which the temperature and coverage dependence of the chemical potential of the adsorbate is given by [32]

$$
\mu_{a}=-V_{0}+k_{\mathrm{B}} T\left[\ln \left(\frac{\theta}{1-\theta}\right)-\ln \left(q_{3} q_{\mathrm{int}}\right)\right],
$$

where $V_{0}$ is the (positive) binding energy of an isolated molecule on the surface. Moreover,

$$
q_{3}=q_{\mathrm{z}} q_{\mathrm{xy}}
$$

is the molecular partition function for an adsorbed molecule where

$$
q_{\mathrm{z}}=\frac{\exp \left(-\frac{h v_{\mathrm{z}}}{2 k_{\mathrm{B}} T}\right)}{1-\exp \left(-\frac{h v_{\mathrm{z}}}{k_{\mathrm{B}} T}\right)}
$$

is the vibrational partition function for motion normal to the surface and $q_{\mathrm{xy}}$ is the partition function for the motion parallel to the surface. If the adsorbate can be treated as a two-dimensional ideal gas, then $q_{\mathrm{xy}}$ can be written as

$$
q_{\mathrm{xy}}=\frac{a_{s}}{\lambda_{\mathrm{th}}^{2}} .
$$

Finally, $q_{\text {int }}$ is the internal partition function of an adsorbed molecule in which some of the internal degrees of freedom are frozen out or frustrated. In the high temperature limit $\left(k_{\mathrm{B}} T \gg h v_{\mathrm{z}}\right)$ for a nonlocalized, noninteracting adsorbate, we can apply these expressions to

$$
R_{\mathrm{d}}=S(\theta, T) \frac{\theta}{1-\theta} \frac{Z_{\text {int }}}{q_{\text {int }}} v_{\mathrm{z}} \exp \left(-\frac{V_{0}}{k_{\mathrm{B}} T}\right) .
$$

In particular, if $S(\theta, T)=1-\theta$, then (A9) becomes the familiar first-order Polanyi-Wigner rate expression:

$$
R_{\mathrm{d}}=\theta \frac{Z_{\text {int }}}{q_{\text {int }}} v_{\mathrm{z}} \exp \left(-\frac{V_{0}}{k_{\mathrm{B}} T}\right) .
$$

We finally note that the above considerations can be generalized to associative desorption [6], e.g., the associative desorption of two adsorbed oxygen atoms to $\mathrm{O}_{2}$. In such a case, we would have that $\mu_{g}^{\mathrm{O}_{2}}=2 \mu_{a}^{\mathrm{O}}$. Using the appropriate expression of the sticking coefficient, $S(\theta, T)=(1-\theta)^{2}$, we would then get the appropriate second-order rate expression for desorption.

\section{Diffusion of PO and $\mathrm{Py}$ on $\mathrm{Cu}(111)$}

To account for translational degrees of freedom for PO and $\mathrm{Py}$ on $\mathrm{Cu}(111)$ in free energy calculations, estimates of their site-to-site diffusion (or hopping) barriers must be obtained. We considered PO, Py, and PyPO cluster diffusion on $\mathrm{Cu}(111)$. All relevant species preferentially adsorb to top sites on $\mathrm{Cu}(111)$, so hopping barriers between adjacent top sites were computed using the climbing-image nudged elastic band method [33]. Results of these calculations for PO and Py are presented in Fig. 5.

The hopping barrier for PO on the surface is $0.11 \mathrm{eV}$ and the hopping barrier for Py on the surface is $0.05 \mathrm{eV}$. The hopping barrier calculation for the PyPO complex returned a value of roughly $5 \mathrm{meV}$, which is too small in magnitude to be trusted at the DFT level. Therefore, the translations of the complex along the surface were considered to be barrierless. It should be noted that intermolecular attractions have led to increased surface mobility of complexes in the literature, so the result of this calculation is not necessarily unexpected [14]. A schematic of the complex diffusion is shown in Fig. 6.

\section{One-dimensional rotor partition function}

In light of the results from a previous section, we will be treating the rotations of isolated PO and Py molecules as effectively barrierless due to the insignificant magnitude of their "rotational barriers." Therefore, to treat these rotations in thermodynamic calculations, the energy levels from the Schrödinger equation must be used. These energy levels parametrized by quantum number $J, E_{\mathrm{rot}, J}$, are

$$
E_{\mathrm{rot}, J}=\frac{h^{2}}{8 \pi^{2} I} J^{2}, \quad \forall J \in \mathbb{Z} .
$$

In the equation above, $I$ is the moment of inertia about the molecule's surface-normal rotational axis (defined as the vector normal to the center of the top site) and $h$ is Planck's constant. The one-dimensional rotational partition function, $q_{\text {rot,1D }}$, is defined as

$$
q_{\mathrm{rot}, 1 \mathrm{D}}=\sum_{J=-\infty}^{\infty} \exp \left(-\frac{E_{\mathrm{rot}, J}}{k_{\mathrm{B}} T}\right) .
$$

Note that $T$ is the absolute temperature and $k_{\mathrm{B}}$ is Boltzmann's constant in the above equation. Inserting the energies from 
(a)
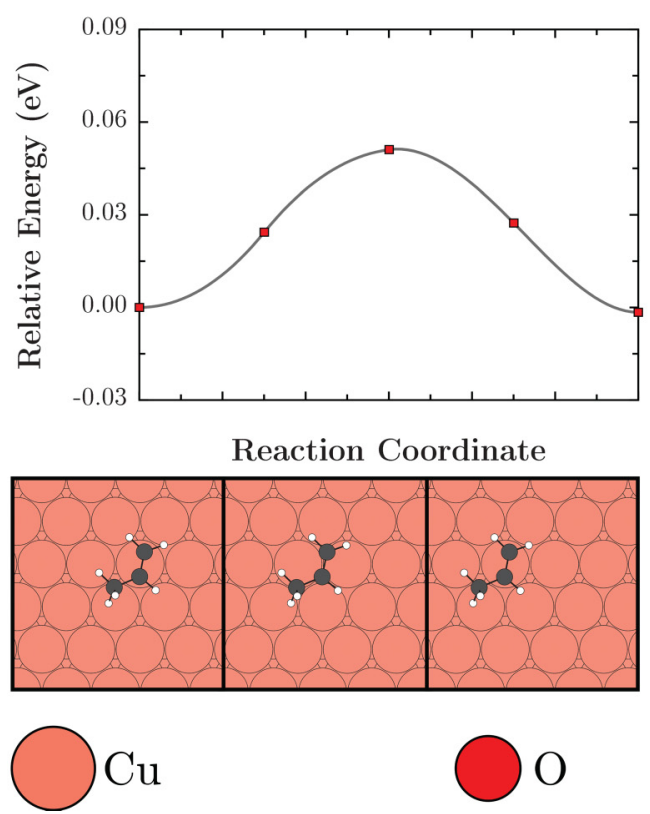

(b)
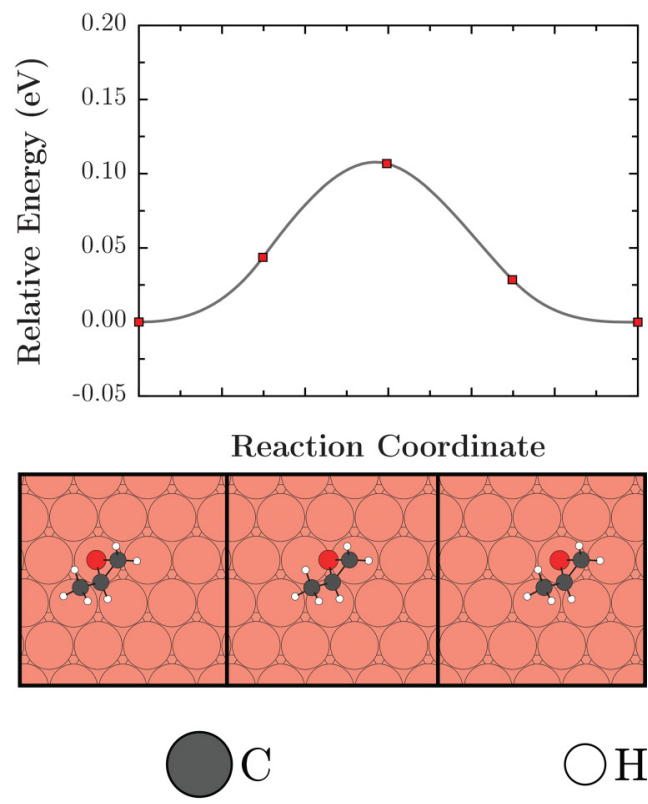

FIG. 5. Minimum energy pathways for propylene (Py) and propylene oxide (PO) between favorable adsorption sites on $\mathrm{Cu}(111)$ with atomistic models of the structures along the pathway.

(A11) into (A12) yields

$$
q_{\mathrm{rot}, 1 \mathrm{D}}=\sum_{J=-\infty}^{\infty} \exp \left(-\frac{J^{2} \Theta_{\mathrm{R}}}{T}\right)
$$

with $\Theta_{\mathrm{R}}$ being the rotational temperature defined as

$$
\Theta_{\mathrm{R}}=\frac{h^{2}}{8 \pi^{2} I k_{\mathrm{B}}} .
$$

If we assume this sum can be approximated to be an integral due to the close spacing of the energy levels, the onedimensional partition function for our molecular rotors is

$$
q_{\mathrm{rot}, 1 \mathrm{D}}=\int_{-\infty}^{\infty} \exp \left(-\frac{J^{2} \Theta_{\mathrm{R}}}{T}\right) d J=\sqrt{\frac{\pi T}{\Theta_{\mathrm{R}}}} .
$$

\section{Chemical potentials of surface-bound species}

In this section, we derive chemical potentials of Py and $\mathrm{PO}$ monomers as well as PyPO clusters on the $\mathrm{Cu}(111)$ surface using fundamental statistical mechanics. We will work in the canonical ensemble, as this is the sensible ensemble for this problem.

\section{a. Chemical potentials of uncomplexed molecules}

We begin by writing the Helmholtz energy for a system of $N_{\mathrm{PO}}$ propylene oxide molecules that are not clustered. Under the assumption that the particles are noninteracting, the partition function for this system, $Q_{\mathrm{PO}}$, would be (accounting for indistinguishability)

$$
Q_{\mathrm{PO}}=\frac{q_{\mathrm{PO}}^{N_{\mathrm{PO}}}}{N_{\mathrm{PO}} !} .
$$

In this equation, $q_{\mathrm{PO}}$ is the microcanonical partition function for PO. Consider the Helmholtz free energy of this ensemble of molecules, $A_{\mathrm{PO}}$, using the standard statistical mechanical definition:

$$
A_{\mathrm{PO}}=-k_{\mathrm{B}} T \ln \left(\frac{q_{\mathrm{PO}}^{N_{\mathrm{PO}}}}{N_{\mathrm{PO}} !}\right) .
$$

Applying Stirling's approximation and rearranging yields

$$
\begin{aligned}
& A_{\mathrm{PO}}=-N_{\mathrm{PO}} k_{\mathrm{B}} T \ln \left(q_{\mathrm{PO}}\right)+k_{\mathrm{B}} T\left(N_{\mathrm{PO}} \ln N_{\mathrm{PO}}-N_{\mathrm{PO}}\right), \\
& A_{\mathrm{PO}}=-N_{\mathrm{PO}} k_{\mathrm{B}} T \ln \left(\frac{q_{\mathrm{PO}}}{N_{\mathrm{PO}}}\right)-N_{\mathrm{PO}} k_{\mathrm{B}} T .
\end{aligned}
$$
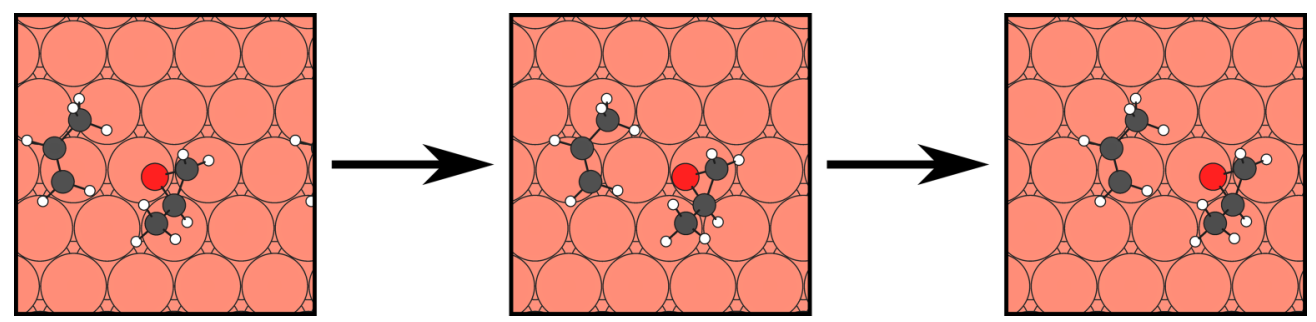

FIG. 6. Schematic of pathway for (approximately) barrierless hopping of the PyPO complex on $\mathrm{Cu}(111)$. 
For the microcanonical partition function, we assume there are electronic, rotational, and translational degrees of freedom. Note that vibrational degrees of freedom were not considered as it was assumed the vibrational degrees of freedom in the complexed and noncomplexed states were approximately identical and that all significant changes in Helmholtz energy would arise due to changes in rotational and translational motion. Rotations of these separate molecules will be treated as barrierless (due to the fact that these molecules rotate at $5 \mathrm{~K}$ and thus have essentially barrierless rotations) [17] and translations will be considered hindered using the results from Sec. 2. The treatment from Sec. 3 will be used for treatment of the rotational partition function for these molecules. The electronic partition function for PO, $q_{\mathrm{elec}, \mathrm{PO}}$, will be (after neglecting excited states)

$$
q_{\text {elec }, \mathrm{PO}}=\exp \left(-\frac{\Delta E_{\mathrm{ads}, \mathrm{PO}}}{k_{\mathrm{B}} T}\right) .
$$

In this equation, $\Delta E_{\text {ads,PO }}$ is the adsorption energy of $\mathrm{PO}$ on the $\mathrm{Cu}(111)$ surface. Note that the energetic zero is taken as the isolated molecule in the gas phase. The hindered translator partition function for a surface-bound species $s, q_{\mathrm{h} \text {-trans, } s}$, is defined as follows [13,31]:

$$
q_{\mathrm{h} \text {-trans }, s}=\frac{N_{s}\left(\frac{\pi r_{x, s}}{T_{x, s}}\right) \exp \left(-\frac{r_{x, s}}{T_{x, s}}\right) \exp \left(-\frac{1}{T_{x, s}}\right) I_{0}^{2}\left(\frac{r_{x, s}}{2 T_{x, s}}\right)}{\left[1-\exp \left(-\frac{1}{T_{x, s}}\right)\right]^{2}},
$$

where $I_{0}$ is the zero-order modified Bessel function of the first kind and $r_{x, s}$ and $T_{x, s}$ are species dependent parameters defined as

$$
\begin{aligned}
r_{x, s} & =\left(\frac{2 E_{a, s} m_{s} a^{2}}{h^{2}}\right)^{\frac{1}{2}}, \\
T_{x, s} & =\frac{k_{\mathrm{B}} T}{h}\left(\frac{2 m_{s} a^{2}}{E_{a, s}}\right)^{\frac{1}{2}} .
\end{aligned}
$$

In these expressions, $N_{s}$ is the number of sites available for diffusion (i.e., number of $\mathrm{Cu}$ atoms), $E_{a, s}$ is the hopping barrier between nearest-neighbor sites on the surface for species $s, m_{s}$ is the mass of the species, and $a$ is the nearest-neighbor site-to-site distance between two surface $\mathrm{Cu}$ atoms. For simplification of notation, we will define $\chi_{s}(T)$, a species-dependent parameter, as

$$
\chi_{s}(T)=\frac{\left(\frac{\pi r_{x, s}}{T_{x, s}}\right) \exp \left(-\frac{r_{x, s}}{T_{x, s}}\right) \exp \left(-\frac{1}{T_{x, s}}\right) I_{0}^{2}\left(\frac{r_{x, s}}{2 T_{x, s}}\right)}{\left[1-\exp \left(-\frac{1}{T_{x, s}}\right)\right]^{2}},
$$

which makes the hindered translator partition function for a species $s$ :

$$
q_{\mathrm{h} \text {-trans,s }}=\chi_{s}(T) N_{s} .
$$

Using all of this, the microcanonical partition function for PO is

$$
\begin{aligned}
q_{\mathrm{PO}} & =q_{\mathrm{elec}, \mathrm{PO}} q_{\mathrm{rot}, 1 \mathrm{D}} q_{\mathrm{h} \text {-trans }, s} \\
& =\exp \left(-\frac{\Delta E_{\mathrm{ads}, \mathrm{PO}}}{k_{\mathrm{B}} T}\right) \sqrt{\frac{\pi T}{\Theta_{\mathrm{R}, \mathrm{PO}}}} \chi_{\mathrm{PO}}(T) N_{s},
\end{aligned}
$$

where $\Theta_{R, P O}$ is the rotational temperature for PO, which is defined in Sec. 3. Using this partition function in (A19), the
Helmholtz free energy of this ensemble is

$A_{\mathrm{PO}}=N_{\mathrm{PO}}\left[\Delta E_{\mathrm{ads}, \mathrm{PO}}-k_{\mathrm{B}} T \ln \left(\sqrt{\frac{\pi T}{\Theta_{\mathrm{R}, \mathrm{PO}}}} \chi_{\mathrm{PO}}(T) \frac{N_{s}}{N_{\mathrm{PO}}}\right)-k_{\mathrm{B}} T\right]$.

If we define the surface coverage of $\mathrm{PO} \theta_{\mathrm{PO}}$ as

$$
\theta_{\mathrm{PO}}=\frac{N_{\mathrm{PO}}}{N_{s}},
$$

the equation for the Helmholtz energy of this ensemble becomes

$$
A_{\mathrm{PO}}=N_{\mathrm{PO}}\left[\Delta E_{\mathrm{ads}, \mathrm{PO}}-k_{\mathrm{B}} T \ln \left(\sqrt{\frac{\pi T}{\Theta_{\mathrm{R}, \mathrm{PO}}}} \frac{\chi_{\mathrm{PO}}(T)}{\theta_{\mathrm{PO}}}\right)-k_{\mathrm{B}} T\right] .
$$

Using this equation, we can define the chemical potential of $\mathrm{PO}$ on the surface, $\mu_{\mathrm{PO}}\left(T, \theta_{\mathrm{PO}}\right)$. From standard thermodynamics, this is defined as

$$
\mu_{\mathrm{PO}}\left(T, \theta_{\mathrm{PO}}\right)=\left(\frac{\delta A_{\mathrm{PO}}}{\delta N_{\mathrm{PO}}}\right)_{T, N_{s}} .
$$

Using this definition and our Helmholtz energy expression for this ensemble of PO molecules (noting that the coverage is a function of $N_{\mathrm{PO}}$ ), the chemical potential is

$$
\begin{aligned}
\mu_{\mathrm{PO}}\left(T, \theta_{\mathrm{PO}}\right)= & \Delta E_{\mathrm{ads}, \mathrm{PO}}-k_{\mathrm{B}} T \ln \left(\sqrt{\frac{\pi T}{\Theta_{\mathrm{R}, \mathrm{PO}}}} \chi_{\mathrm{PO}}(T)\right) \\
& +k_{\mathrm{B}} T \ln \left(\theta_{\mathrm{PO}}\right) .
\end{aligned}
$$

This derivation can be exactly mirrored for an ensemble of $N_{\text {Py }}$ molecules on the surface that have equivalent statisticalmechanical degrees of freedom (i.e., they are free rotors, hindered translators, and only have a ground electronic state). Thus, if we define the coverage of Py as

$$
\theta_{\mathrm{Py}}=\frac{N_{\mathrm{Py}}}{N_{s}}
$$

and let all other variables for Py mirror those in the PO derivation above, the chemical potential of $\mathrm{Py}, \mu_{\mathrm{Py}}\left(T, \theta_{\mathrm{PO}}\right)$, on the surface can be written as

$$
\begin{aligned}
\mu_{\mathrm{Py}}\left(T, \theta_{\mathrm{Py}}\right)= & \Delta E_{\mathrm{ads}, \mathrm{Py}}-k_{\mathrm{B}} T \ln \left(\sqrt{\frac{\pi T}{\Theta_{\mathrm{R}, \mathrm{Py}}}} \chi_{\mathrm{Py}}(T)\right) \\
& +k_{\mathrm{B}} T \ln \left(\theta_{\mathrm{Py}}\right) .
\end{aligned}
$$

\section{b. Chemical potential of PyPO complexes}

We now consider an ensemble of $N_{\text {PyPO }}$ complexes on the surface. To find the chemical potential of a complex on the surface, we must first determine the Helmholtz energy of this ensemble of complexes. Considering each complex as an individual, indistinguishable entity, the canonical partition function for this system, $Q_{\mathrm{PyPO}}$, would be

$$
Q_{\mathrm{PyPO}}=\frac{q_{\mathrm{PyPO}}^{N_{\mathrm{PyO}}}}{N_{\mathrm{PyPO}} !},
$$


where $q_{\mathrm{PyPO}}$ is the microcanonical partition function of a PyPO complex. The Helmholtz free energy of an ensemble of $N_{\mathrm{PyPO}}$ complexes would be

$$
A_{\mathrm{PyPO}}=-N_{\mathrm{PyPO}} k_{\mathrm{B}} T \ln \left(\frac{q_{\mathrm{PyPO}}}{N_{\mathrm{PyPO}}}\right)-N_{\mathrm{PyPO}} k_{\mathrm{B}} T .
$$

In terms of degrees of freedom, these clusters are treated as free translators given the negligible diffusion barriers computed in Sec. 2. Further, they are rotationally arrested according to experimental STM images and thus possess no rotational degrees of freedom. Finally, they do possess an electronic degree of freedom with an electronic partition function, $q_{\mathrm{elec}, \mathrm{PyPO}}$, that is given as follows:

$$
q_{\text {elec,PyPO }}=\exp \left(-\frac{\Delta E_{\mathrm{ads}, \mathrm{PyPO}}}{k_{\mathrm{B}} T}\right) .
$$

In this equation, $\Delta E_{\mathrm{ads}, \mathrm{PyPO}}$ is the adsorption energy of the PyPO complex (with respect to the isolated species in the gas phase). Again, the use of the complex adsorption energy indicates that our energetic zero is the isolated molecules in the gas phase. The neglect of vibrational degrees of freedom is discussed above. The free translator partition function, $q_{\text {trans, } \mathrm{PyPO}}$, is

$$
q_{\text {trans, } \mathrm{PyPO}}=\frac{2 \pi m_{\mathrm{PyPO}} k_{\mathrm{B}} T}{h^{2}} N_{s}\left(\frac{\sqrt{3}}{2} a^{2}\right),
$$

where the area available for translation is expressed based on the number of sites on the $\mathrm{Cu}(111)$ surface and the area of a surface unit cell $\left(\sqrt{3} a^{2} / 2\right)$. Using both of these partition functions, the microcanonical partition function for the PyPO complex is

$$
\begin{aligned}
q_{\mathrm{PyPO}} & =q_{\text {elec,PyPO }} q_{\text {trans }, \mathrm{PyPO}} \\
& =\exp \left(-\frac{\Delta E_{\mathrm{ads}, \mathrm{PyPO}}}{k_{\mathrm{B}} T}\right) \frac{2 \pi m_{\mathrm{PyPO}} k_{\mathrm{B}} T}{h^{2}} N_{s}\left(\frac{\sqrt{3}}{2} a^{2}\right) .
\end{aligned}
$$

Defining the coverage of the complex, $\theta_{\mathrm{PyPO}}$, as

$$
\theta_{\mathrm{PyPO}}=\frac{N_{\text {PyPO }}}{N_{s}},
$$

the Helmholtz energy of this ensemble of complexes can be written using (A35) and (A37):

$$
\begin{aligned}
A_{\mathrm{PyPO}}= & N_{\mathrm{PyPO}}\left\{\Delta E_{\mathrm{ads}, \mathrm{PyPO}}-k_{\mathrm{B}} T\right. \\
& \left.\times \ln \left[\frac{2 \pi m_{\mathrm{PyPO}} k_{\mathrm{B}} T}{h^{2}} \frac{1}{\theta_{\mathrm{PyPO}}}\left(\frac{\sqrt{3}}{2} a^{2}\right)\right]-k_{\mathrm{B}} T\right\} .
\end{aligned}
$$

We can calculate the chemical potential of a PyPO complex, $\mu_{\mathrm{PyPO}}\left(T, \theta_{\mathrm{PyPO}}\right)$, on the surface using an equation analogous to (A30):

$$
\begin{aligned}
\mu_{\mathrm{PyPO}}\left(T, \theta_{\mathrm{PyPO}}\right)= & \Delta E_{\text {ads, } \mathrm{PyPO}} \\
& -k_{\mathrm{B}} T \ln \left[\frac{2 \pi m_{\mathrm{PyPO}} k_{\mathrm{B}} T}{h^{2}}\left(\frac{\sqrt{3}}{2} a^{2}\right)\right] \\
& +k_{\mathrm{B}} T \ln \left(\theta_{\mathrm{PyPO}}\right) .
\end{aligned}
$$

\section{Experimental and computational methods}

LT-STM experiments were carried out in an UHV chamber with background pressure of $1 \times 10^{-11}$ mbar using an Omicron Nanotechnology low-temperature scanning tunneling microscope. The crystal was cleaned by cycles of $\mathrm{Ar}^{+}$ sputtering and thermal annealing in an adjoining preparation chamber with a base pressure of $2 \times 10^{-10}$ mbar. Adsorbates were deposited on a clean $\mathrm{Cu}(111)$ crystal at $5 \mathrm{~K}$ using a precision leak valve and the surface was briefly annealed to greater than $40 \mathrm{~K}$ to equilibrate the adsorbates. Propylene and propylene oxide samples were procured from Matheson Tri-gas and Sigma Aldrich, respectively. Propylene (99.9\% purity) was used as received and propylene oxide samples (99.9\% purity) were subjected to multiple freeze-pump-thaw cycles. All imaging was performed at $5 \mathrm{~K}$. Typical imaging conditions were between 60 and $-60 \mathrm{mV}$ with a tunneling current less than $70 \mathrm{pA}$.

TPD experiments were conducted in a separate UHV chamber with a background pressure less than $1 \times 10^{-10} \mathrm{mbar}$. Experiments were conducted on a $\mathrm{Cu}(111)$ surface which was cleaned with repeated $\mathrm{Ar}^{+}$sputtering with an RBD hot filament sputter gun and thermal annealing to $750 \mathrm{~K}$. Propylene and propylene oxide were dosed using a precision leak valve while the crystal was held at $85 \mathrm{~K}$. The chamber was equipped with a shielded Hiden Hal RC 201 mass spectrometer capable of being advanced to within less than $1 \mathrm{~mm}$ of the crystal surface for TPD experiments. Only reversible desorption was observed so cleaning was only done at the start of each day of experiments and controls were performed to check that the same results were obtained on a freshly cleaned crystal. The crystal was heated via resistive heating and cooled via liquid nitrogen. Temperatures were measured with a $K$-type thermocouple. Experiments were conducted with a linear heating rate of $1.5 \mathrm{~K} / \mathrm{s}$ and TPD traces are background subtracted and corrected for ionization cross section, ionization pattern, and relative sensitivity of the mass spectrometer at the specific $m / z$ of interest. Coverages are reported relative to the number of $\mathrm{Cu}$ atoms unless otherwise noted. Comparison of desorption of a saturated monolayer of both Py and PO revealed a similar packing density, with $\sim 1$ molecule per $5 \mathrm{Cu}$ atoms. We calculated these values based on comparison of the corrected area with the saturation desorption feature of $\mathrm{CO}$, which has a known packing density of $0.52 \mathrm{CO}$ per $\mathrm{Cu}$ atom [34]. In terms of chirality we used primarily R-PO for these experiments but trials with S-PO gave the same desorption energies as expected.

All theoretical calculations were performed using closedshell density functional theory implemented within the Vienna $a b$ initio simulation package (VASP), which calculates the electronic energy of a periodic system using a plane-wave basis set $[35,36]$. These energies were computed using the frozen-core approximation for treatment of the core electrons along with the projector augmented wave method to quench valence wave function oscillations near core regions [37,38]. The data sets used to implement this approach were released by VASP developers in 2012. A cutoff energy of $500 \mathrm{eV}$ was used to determine the number of plane waves employed at each $k$ point. The Perdew-Burke-Ernzerhof (PBE) functional was used to treat electronic exchange and correlation [39]. 
Additionally, a $3 \times 3 \times 1$ Monkhorst-Pack grid was used to sample the first Brillouin zone. Using these quantum mechanical parameters, geometry optimizations were performed using a conjugate-gradient algorithm and transition states were obtained using the climbing-image nudged elastic band (CINEB) method $[33,40]$. Transition state structures were confirmed to have a single imaginary vibrational frequency through standard harmonic analysis performed with in-house software. These calculations were considered converged once appropriate atomic forces were lower than $0.02 \mathrm{eV} / \AA$. A $(4 \times 4) \mathrm{Cu}(111)$ supercell (four layers in thickness; theoretical lattice constant $3.635 \AA$ ) was used for all presented calculations. This lattice constant was obtained using a primitive face-centered cubic unit cell where a $12 \times 12 \times 12 k$-point Monkhorst-Pack grid was used to sample the first Brillouin zone. In this cell, the bottom two layers were held fixed in bulk positions during geometry optimizations and transition state calculations. The vacuum thickness between periodic metallic slabs was at least $15 \AA$ in all cases.

In the text, we use the barrier calculations presented in the supplemental information and electronic complexation energies from work by Sykes and co-workers to compute the free energy change of complexation [17]. The electronic portion of the complexation free energy was calculated using a generalized gradient approximation functional that included dispersive corrections (the optB88-vdw functional) while the functional used to compute the barriers in this work did not include corrections of this nature. As different functionals have been shown to produce relatively similar reaction energies (and thus likely similar activation energies), the mixing of these functionals in our free energy calculation is justified [41].
[1] G. A. Somorjai and Y. Li, Impact of surface chemistry, Proc. Natl. Acad. Sci. USA 108, 917 (2010).

[2] J. K. Nørskov, F. Abild-Pedersen, F. Studt, and T. Bligaard, Density functional theory in surface chemistry and catalysis, Proc. Natl. Acad. Sci. USA 108, 937 (2011).

[3] A. Vojvodic and J. K. Nørskov, New design paradigm for heterogeneous catalysts, Natl. Sci. Rev. 2, 140 (2015).

[4] J. K. Nørskov, T. Bligaard, B. Hvolbæk, F. Abild-Pedersen, I. Chorkendorff, and C. H. Christensen, The nature of the active site in heterogeneous metal catalysis, Chem. Soc. Rev. 37, 2163 (2008).

[5] H. J. Kreuzer, S. H. Payne, A. Drozdowski, and D. Menzel, Theory of dissociative and nondissociative adsorption and desorption, J. Chem. Phys. 110, 6982 (1999).

[6] H. J. Kreuzer and S. H. Payne, Theories of adsorptiondesorption kinetics on homogeneous surfaces, Stud. Surf. Sci. Catal. 104, 153 (1997).

[7] J. F. Weaver, Entropies of adsorbed molecules exceed expectations, Science 339, 39 (2013).

[8] C. T. Campbell, L. Árnadóttir, and J. R. Sellers, Kinetic prefactors of reactions on solid surfaces, Z. Phys. Chem. 227, 1435 (2013).

[9] C. T. Campbell and J. R. Sellers, The entropies of adsorbed molecules, J. Am. Chem. Soc. 134, 18109 (2012).

[10] H. Hedgeland, M. Sacchi, P. Singh, A. J. McIntosh, A. P. Jardine, G. Alexandrowicz, D. J. Ward, S. J. Jenkins, W. Allison, and J. Ellis, Mass transport in surface diffusion of Van der Waals bonded systems: Boosted by rotations? J. Phys. Chem. Lett. 7, 4819 (2016).

[11] J. C. Gehrig, M. Penedo, M. Parschau, J. Schwenk, M. A. Marioni, E. W. Hudson, and H. J. Hug, Surface singlemolecule dynamics controlled by entropy at low temperatures, Nat. Commun. 8, 14404 (2017).

[12] C. T. Campbell, L. H. Sprowl, and L. Árnadóttir, Equilibrium constants and rate constants for adsorbates: Two-dimensional (2D) ideal gas, 2D ideal lattice gas, and ideal hindered translator models, J. Phys. Chem. C 120, 10283 (2016).

[13] L. H. Sprowl, C. T. Campbell, and L. Árnadóttir, Hindered translator and hindered rotor models for adsorbates: partition functions and entropies, J. Phys. Chem. C 120, 9719 (2016).
[14] B. G. Briner, M. Doering, H.-P. Rust, and A. M. Bradshaw, Microscopic molecular diffusion enhanced by adsorbate interactions, Science 278, 257 (1997).

[15] S. H. Payne, J.-S. McEwen, H. J. Kreuzer, and D. Menzel, Adsorption and desorption of $\mathrm{CO}$ on $\mathrm{Ru}(0001)$ : A comprehensive analysis, Surf. Sci. 594, 240 (2005).

[16] J.-S. McEwen, S. H. Payne, H. J. Kreuzer, M. Kinne, R. Denecke, and H.-P. Steinrück, Adsorption and desorption of $\mathrm{CO}$ on Pt(111): A comprehensive analysis, Surf. Sci. 545, 47 (2003).

[17] A. M. Larson, K. Groden, R. T. Hannagan, J.-S. McEwen, and E. C. H. Sykes, Understanding enantioselective interactions by pulling apart molecular rotor complexes, ACS Nano 13, 5939 (2019).

[18] P. A. Redhead, Thermal desorption of gases, Vacuum 12, 203 (1962).

[19] J. Chao and B. J. Zwolinski, Ideal gas thermodynamic properties of ethylene and propylene, J. Phys. Chem. Ref. Data 4, 251 (1975).

[20] J. Chao, K. R. Hall, K. N. Marsh, and R. C. Wilhoit, Thermodynamic properties of key organic oxygen compounds in the carbon range $\mathrm{C} 1$ to $\mathrm{C} 4$. Part 2. Ideal gas properties, J. Phys. Chem. Ref. Data 15, 1369 (1986).

[21] J. D. Horvath and A. J. Gellman, Enantiospecific desorption of chiral compounds from chiral $\mathrm{Cu}(643)$ and achiral $\mathrm{Cu}(111)$ surfaces, J. Am. Chem. Soc. 124, 2384 (2002).

[22] J. D. Horvath and A. J. Gellman, Enantiospecific desorption of $\mathrm{R}$ - and S-propylene oxide from a chiral $\mathrm{Cu}(643)$ surface, J. Am. Chem. Soc. 123, 7953 (2001).

[23] J. M. Meyers and A. J. Gellman, The investigation of uorinated propenes on the $\mathrm{Cu}(111)$ surface, Surf. Sci. 339, 57 (1995).

[24] S. C. Street and A. J. Gellman, Orientation of physisorbed uoropropenes on $\mathrm{Cu}(111)$, J. Phys. Chem. B 101, 1389 (2002).

[25] W. X. Huang and J. M. White, Propene adsorption on $\operatorname{Ag}(111)$ : A TPD and RAIRS study, Surf. Sci. 513, 399 (2002).

[26] K. Golibrzuch, P. R. Shirhatti, J. Geweke, J. Werdecker, A. Kandratsenka, D. J. Auerbach, A. M. Wodtke, and C. Bartels, $\mathrm{CO}$ desorption from a catalytic surface: Elucidation of the role of steps by velocity-selected residence time measurements, J. Am. Chem. Soc. 137, 1465 (2015). 
[27] B. Tränkenschuh, N. Fritsche, T. Fuhrmann, C. Papp, J. F. Zhu, R. Denecke, and H. P. Steinrück, A site-selective in situ study of CO adsorption and desorption on Pt(355), J. Chem. Phys. 124, 074712 (2006).

[28] J.-S. McEwen, S. H. Payne, H. J. Kreuzer, and C. Bracher, Hopping kinetics on a finite 1D chain: An exact analysis, Int. J. Quantum Chem. 106, 2889 (2006).

[29] K. L. Kostov, M. Gsell, W. Widdra, S. H. Payne, H. J. Kreuzer, and D. Menzel, Lateral interactions and nonequilibrium in adsorption and desorption. Part 1. Experimental results for $(2 \times 2)-(3 \mathrm{O}+\mathrm{NO}) / \mathrm{Ru}(001)$, Surf. Sci. 600, 4650 (2006).

[30] S. H. Payne, J.-S. McEwen, H. J. Kreuzer, and D. Menzel, Lateral interactions and nonequilibrium in adsorption and desorption. Part 2. A kinetic lattice gas model for $(2 \times 2)-(3 \mathrm{O}+\mathrm{NO}) /$ Ru(001), Surf. Sci. 600, 4660 (2006).

[31] T. L. Hill, An Introduction to Statistical Thermodynamics (Dover Publications, Mineola, NY, 2012).

[32] H. Kreuzer and S. H. Payne, Theories of the adsorptiondesorption kinetics on homogeneous surfaces, in Equilibria and Dynamics of Gas Adsorption on Heterogeneous Solid Surfaces, edited by W. Rudziński, W. Steele, and G. Zgrablich, Studies in Surface Science and Catalysis Vol. 104 (Elsevier, Amsterdam, 1997), Chap. 3, pp. 153-200.

[33] G. Henkelman, B. P. Uberuaga, and H. Jónsson, A climbing image nudged elastic band method for finding saddle points and minimum energy paths, J. Chem. Phys. 113, 9901 (2000).

[34] M. D. Marcinkowski, A. D. Jewell, M. Stamatakis, M. B. Boucher, E. A. Lewis, C. J. Murphy, G. Kyriakou, and E. C. H. Sykes, Controlling a spillover pathway with the molecular cork effect, Nat. Mater. 12, 523 (2013).

[35] G. Kresse and J. Furthmüller, Efficient iterative schemes for $a b$ initio total-energy calculations using a plane-wave basis set, Phys. Rev. B 54, 11169 (1996).

[36] G. Kresse and J. Hafner, Ab initio molecular dynamics for liquid metals, Phys. Rev. B 47, 558 (1993).

[37] G. Kresse and D. Joubert, From ultrasoft pseudopotentials to the projector augmented-wave method, Phys. Rev. B 59, 1758 (1999).

[38] P. E. Blöchl, Projector augmented-wave method, Phys. Rev. B 50, 17953 (1994).

[39] J. P. Perdew, K. Burke, and M. Ernzerhof, Generalized Gradient Approximation Made Simple, Phys. Rev. Lett. 77, 3865 (1996).

[40] G. Henkelman and H. Jónsson, Improved tangent estimate in the nudged elastic band method for finding minimum energy paths and saddle points, J. Chem. Phys. 113, 9978 (2000).

[41] A. J. R. Hensley, Y. Wang, and J.-S. McEwen, Phenol deoxygenation mechanisms on $\mathrm{Fe}(110)$ and $\mathrm{Pd}(111)$, ACS Catal. 5, 523 (2015). 